\title{
Impulse radar imaging system for concealed object detection
}

DOI:

$10.1117 / 12.2029462$

Link to publication record in Manchester Research Explorer

\section{Citation for published version (APA):}

Podd, F. J. W., David, M., Iqbal, G., Hussain, F., Morris, D., Osakue, E., Yeow, Y., Zahir, S., Armitage, D. W., \& Peyton, A. J. (2013). Impulse radar imaging system for concealed object detection. In Proceedings of SPIE - The International Society for Optical Engineering/Proc SPIE Int Soc Opt Eng (Vol. 8900) https://doi.org/10.1117/12.2029462

Published in:

Proceedings of SPIE - The International Society for Optical Engineering|Proc SPIE Int Soc Opt Eng

\section{Citing this paper}

Please note that where the full-text provided on Manchester Research Explorer is the Author Accepted Manuscript or Proof version this may differ from the final Published version. If citing, it is advised that you check and use the publisher's definitive version.

\section{General rights}

Copyright and moral rights for the publications made accessible in the Research Explorer are retained by the authors and/or other copyright owners and it is a condition of accessing publications that users recognise and abide by the legal requirements associated with these rights.

\section{Takedown policy}

If you believe that this document breaches copyright please refer to the University of Manchester's Takedown Procedures [http://man.ac.uk/04Y6Bo] or contact uml.scholarlycommunications@manchester.ac.uk providing relevant details, so we can investigate your claim.

\section{OPEN ACCESS}




\title{
Impulse radar imaging system for concealed object detection
}

\author{
F.J.W.Podd*, M.David, G.Iqbal, F.Hussain, D. Morris, E.Osakue, Y.Yeow, S.Zahir, \\ D.W.Armitage, A.J.Peyton \\ School of Electrical and Electronic Engineering, University of Manchester, UK
}

\begin{abstract}
Electromagnetic systems for imaging concealed objects at checkpoints typically employ radiation at millimetre and terahertz frequencies. These systems have been shown to be effective and provide a sufficiently high resolution image. However there are difficulties and current electromagnetic systems have limitations particularly in accurately differentiating between threat and innocuous objects based on shape, surface emissivity or reflectivity, which are indicative parameters. In addition, water has a high absorption coefficient at millimetre wavelength and terahertz frequencies, which makes it more difficult for these frequencies to image through thick damp clothing.
\end{abstract}

This paper considers the potential of using ultra wideband (UWB) in the low gigahertz range. The application of this frequency band to security screening appears to be a relatively new field. The business case for implementing the UWB system has been made financially viable by the recent availability of low-cost integrated circuits operating at these frequencies. Although designed for the communication sector, these devices can perform the required UWB radar measurements as well.

This paper reports the implementation of a 2 to $5 \mathrm{GHz}$ bandwidth linear array scanner. The paper describes the design and fabrication of transmitter and receiver antenna arrays whose individual elements are a type of antipodal Vivaldi antenna. The antenna's frequency and angular response were simulated in CST Microwave Studio and compared with laboratory measurements.

The data pre-processing methods of background subtraction and deconvolution are implemented to improve the image quality. The background subtraction method uses a reference dataset to remove antenna crosstalk and room reflections from the dataset. The deconvolution method uses a Wiener filter to "sharpen" the returned echoes which improves the resolution of the reconstructed image. The filter uses an impulse response reference dataset and a signal-to-noise parameter to determine how the frequencies contained in the echo dataset are normalised. The chosen image reconstruction algorithm is based on the back-projection method. The algorithm was implemented in MATLAB and uses a pre-calculated sensitivity matrix to increase the computation speed.

The results include both 2D and 3D image datasets. The 3D datasets were obtained by scanning the dual sixteen element linear antenna array over the test object. The system has been tested on both humans and mannequin test objects. The front surface of an object placed on the human/mannequin torso is clearly visible, but its presence is also seen from a tell-tale imaging characteristic. This characteristic is caused by a reduction in the wave velocity as the electromagnetic radiation passes through the object, and manifests as an indentation in the reconstructed image that is readily identifiable. The prototype system has been shown to easily detect a $12 \mathrm{~mm}$ x $30 \mathrm{~mm}$ x70 mm plastic object concealed under clothing. 


\section{INTRODUCTION}

An important feature of border and security checkpoints is to detect contraband, such as drugs and weapons. Terrorist threats have evolved from checked baggage, to carry-on baggage, to air cargo, and to non-metallic explosives hidden on the body [1]. Cargo and baggage can be evaluated using ionising radiation, whereas humans must be subjected to only low energy and low radiation levels, if it is permitted at all.

Since the human body is a non-metallic object, and the person can be asked to remove metallic objects, metal detectors do not need to discriminate between object types; they only need to signal that a metallic object is present. Of course, non-metallic objects will not be found by the metal detectors, so an alternative means of detecting these objects is required. Consequently, whole body imaging (WBI) techniques are of interest whereby a 3D dataset of the candidate subject is captured to reveal non-metallic objects concealed under clothing. This 3D dataset is required for the purpose of object discrimination by comparison of the measurement body profile with a typical human body shape. Typically millimetric waves or X-rays have been used and the candidate is required to stand with their arms raised, and legs separated, so that the interrogating field (X-ray or electromagnetic) is able to cover the full surface area of the body. The current scanning times for the millimetric wave systems are the order of seconds [1].

To ensure privacy, the 3D body image may be analysed by a computer algorithm rather than by a human operator. This algorithm is termed Automatic Target Recognition (ATR) and presents a simple output to the operator. For example, the user interface may either display the word "OK" when no anomaly is detected, or show an image of the anomaly on a generic outline of a person when a positive alarm occurs [1]

The ideal WBI imaging system for detecting threat objects would have the following features. It would:

- Be safe.

- Be capable of detecting all threat objects such as metal and ceramic knives, IED vests, powdered and liquid drugs etc.

- Make it hard to cloak the threat object e.g. cloaking by embedding it in another object.

- Allow a high number of people to be scanned per minute, preferably a scan time of 15 seconds or less per person [2].

- Be unobtrusive to the person and keep them at their ease, for instance the person simply walks through a corridor.

- Have low false alarm rate whilst having a high detection rate.

- Be able to discriminate between different types of objects to permit the person to walk through without having to put their items in a tray.

In 2013, in the USA there are more than 740 Advanced Imaging Technology machines located at almost 160 airports nationwide [3]. The AIT scanners are also present in governmental locations such as courts, a justice centre and a prison. Both active and passive mm-wave and $\mathrm{THz}$ systems have been deployed with some success in both portal format (walking through a deep door frame), and stand-off detection where the system is a few meters or more from the target. A possible problem with Millimetric wave and Terahertz frequencies is that they are partially absorbed when passing through thick or damp clothing [4] which may lead to a reduction in the signal to noise level. The peak dielectric loss of pure water at $20^{\circ} \mathrm{C}$ is approximately $16 \mathrm{GHz}$, with further resonances in the $100 \mathrm{GHz}$ and terahertz region [5].

The reconstructed images can appear to show artefacts, for instance between the person's legs. This problem is due to multipath propagation of the waves. A typical multipath example is when the emitted EM wave hits a leg, and is then reflected onto the other leg, and from there reflected into the receiving antenna. If the reconstruction method assumes all reflections have come from a single surface reflection then this "second order" reflection echo will be seen as an imaging artefact. It may be possible to remove this multipath artefact using a higher order reconstruction algorithm [6]. In order to detect low reflectivity objects in the presence of strong multipath signals, the reconstruction algorithm needs to model the multipath signals correctly; this would prevent the strong signal from remaining in pixels where there is no object. This would also enable a high reconstructed image dynamic range.

In their November 2011 statement [7], the Transportation Security Administration acknowledged that improvements can be made in detection efficiency in the operation of millimetre wave imaging systems. The research reported here considers the potential of using ultra wideband microwave techniques. 


\section{BACKGROUND - ULTRA WIDE-BAND IMAGING}

There are many electromagnetic methods of object detection which are categorised by their approximate operating frequency bands. Most systems can be operated in an "active" mode where a transducer emits a signal, which then interacts with the object, and is subsequently detected by a receiver. Some systems can also work in a passive mode, relying on signals generated either by the body, or by independent external sources:

- Magnetic: $100 \mathrm{~Hz}$ to $10 \mathrm{kHz}$.

- Capacitive: $100 \mathrm{kHz}$ to $10 \mathrm{MHz}$.

- Millimetric: $30 \mathrm{GHz}$ to $100 \mathrm{GHz}$.

- Terahertz - $100 \mathrm{GHz}$ to $300 \mathrm{GHz}$.

- Infrared.

- X-Ray Backscatter - Currently forbidden in the EU [8], and not used in the USA [9]. There are also non-electromagnetic approaches, for example using ultrasound [10].

The Defence Advanced Research Project Agency (DARPA) 1990 “Assessment of UWB Radar” defined UWB radar as any radar whose fractional bandwidth is greater than 0.25 , regardless of the centre frequency or the signal timebandwidth product [11]. In this paper we use the label "UWB" to mean the band in the low GHz that is licensed for UWB communications by different countries. This band is typically defined as running from $1 \mathrm{GHz}$ to $12 \mathrm{GHz}$. The amount of power that can be emitted at each frequency varies across the band. This permitted power output variation is called the "mask". Many countries define their mask differently. The simplest mask was Singapore's 2003 "UWB Friendly Zone" with a constant power output $(-35 \mathrm{dBm} / \mathrm{MHz})$ over the $2.2 \mathrm{GHz}$ to $10.6 \mathrm{GHz}$ band [12], although Singapore now has a more complicated mask [13].

The low GHz spectrum (e.g. 3 - $10 \mathrm{GHz}$ ) has so far seen little use in security imaging applications. This may be due to it having longer wavelengths $(3 \mathrm{~cm}$ to $10 \mathrm{~cm}$ ) than millimetre waves so from Rayleigh's criterion for angular (cross-range) resolution, for the same array size UWB should have a lower cross-range resolution than millimetre waves. However, the down-range resolution is not limited by signal frequency, but instead it is a function of phase accuracy, and bandwidth for depth disambiguation. A more detailed consideration of resolution is given by [14]. Although it may be easier to obtain a wide absolute bandwidth at a high centre frequency than at a low one, it is possible and practicable to achieve a $5 \mathrm{GHz}$ bandwidth with a centre frequency of about $4 \mathrm{GHz}$.

Threat objects concealed on the body have a constrained size range - they have to be big enough to pose a threat, but small and especially thin enough so that they are not easy to spot under the clothing. UWB wavelengths are of the order of centimetres up to tens of centimetres. The fact that the wavelengths are of the order of the sizes of concealed objects means that the wave interacts with the object as a whole, rather than having an optical type surface reflection. For example, [15] looked at the interaction of UWB and handguns. This interaction may enable object detection using the frequency spectra signature of the echo response in addition to the millimetre wave image processing based object characterisation.

The business case for implementing the UWB system has been made financially viable by the recent availability of lowcost integrated circuits. Although designed for the communication sector, these chips can perform the required radar measurements. 


\section{EXPERIMENTAL SYSTEM}

The system used the Novelda NVA6100A radar integrated circuit for its UWB radar. The system configuration was implemented as shown in figure 1, with the measured capabilities and key parameters of the system given in table 1.

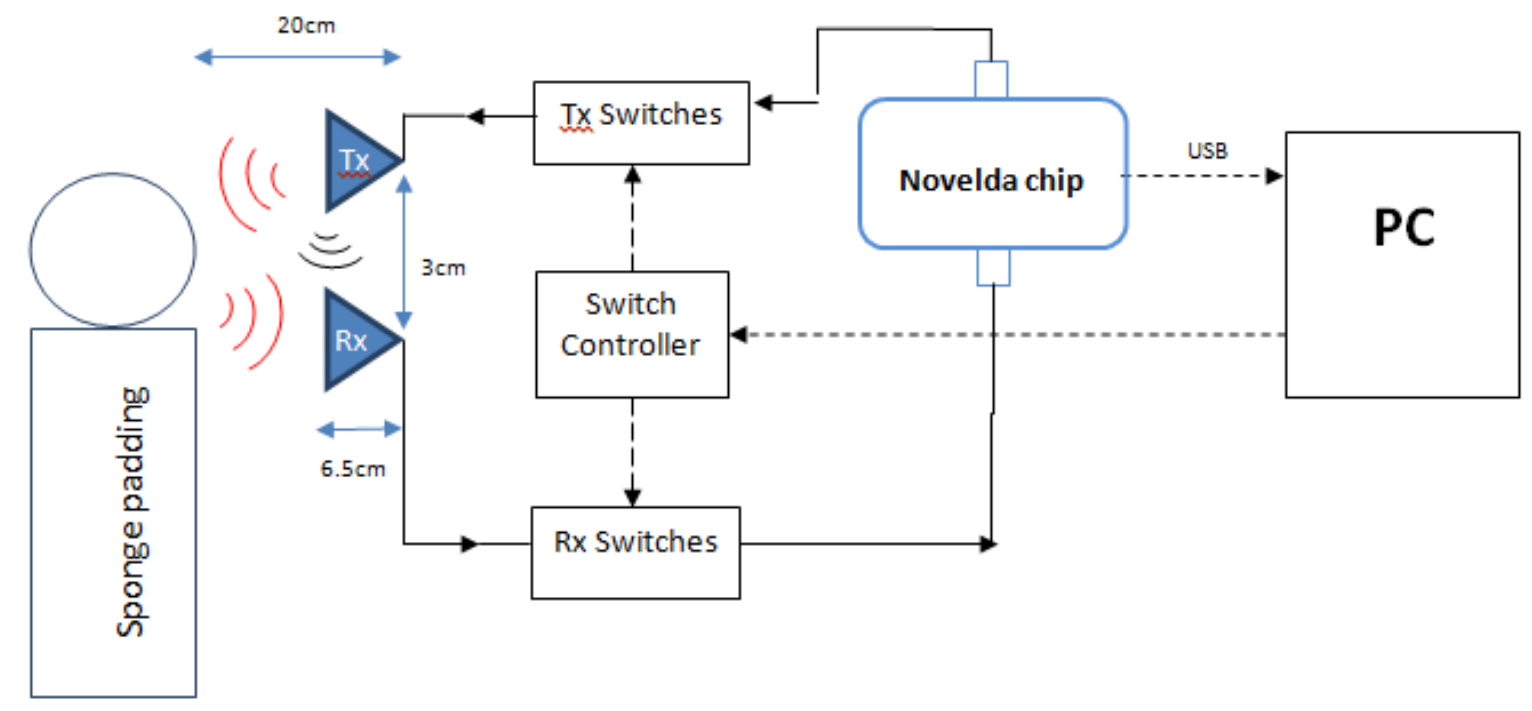

Figure 1: Imaging system block diagram.

\begin{tabular}{|l|l|}
\hline Sampling frequency & $34 \mathrm{GHz}$ \\
\hline Data capture time & $0.2 \mathrm{~s}$ \\
\hline Calculated time to capture for $2 \times 16$ array & $51.2 \mathrm{~s}$ \\
\hline Signal to noise ratio & $40 \mathrm{~dB}$ \\
\hline Spatial resolution & $1 \mathrm{~mm}$ \\
\hline Smallest detectable object (radius) & $8.9 \mathrm{~mm}$ \\
\hline Smallest detectable threat object & $6 \mathrm{~mm}$ \\
\hline Cost (approx). & $£ 400$ \\
\hline Physical size & $0.1 \mathrm{~m} \times 0.1 \mathrm{~m} \times 0.1 \mathrm{~m}$ \\
\hline
\end{tabular}

Table 1: Capabilities and key parameters of the imaging system based on the Novelda module.

The frequency response of the Novelda system was measured using three different input amplifier gain settings and the results are shown in figure 2 . 


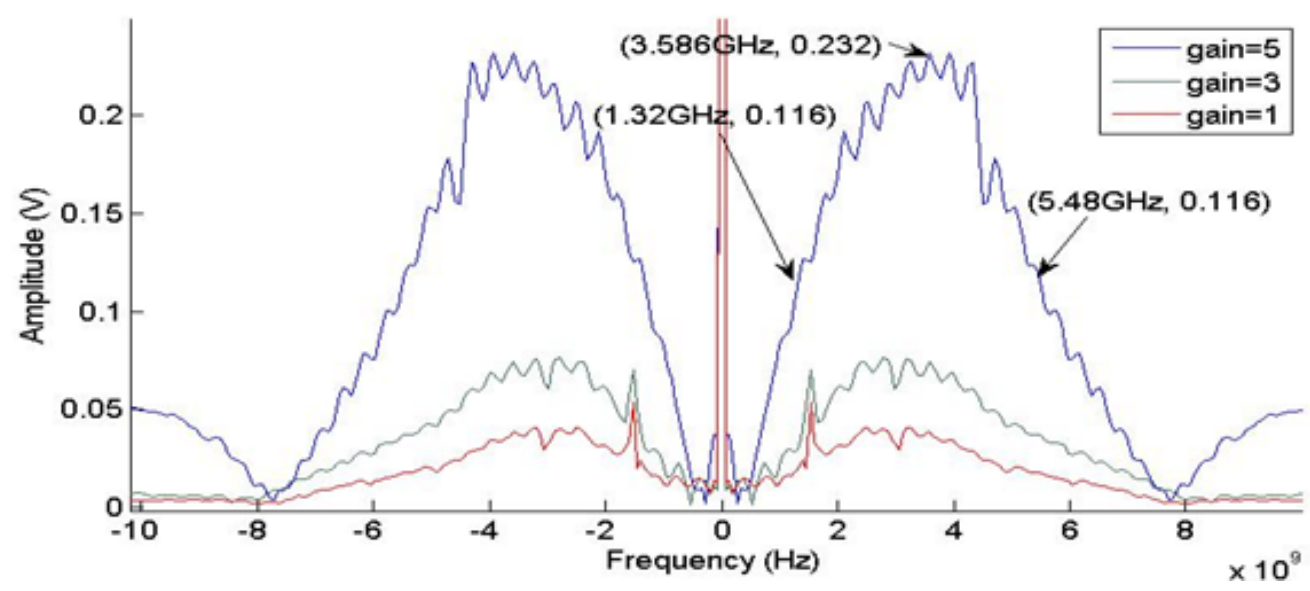

Figure 2: Frequency response of the Novelda radar system for three gain settings.

A total of 32 antennas were chosen for the antenna array. Due to the number of antennas used, desired attributes for antennas in this system are to be lightweight and cheap to manufacture. The antennas should also have a high gain in the boresight direction in order to give a good signal to noise level and to make the system less sensitive to unwanted echoes in other directions. The antipodal Vivaldi antenna was chosen as it measured up to these design requirements.

Previous work in the group was based on Vivaldi antennas. The variation in the S11 frequency response of four of these identically manufactured Vivaldi antennas is shown in figure 3.

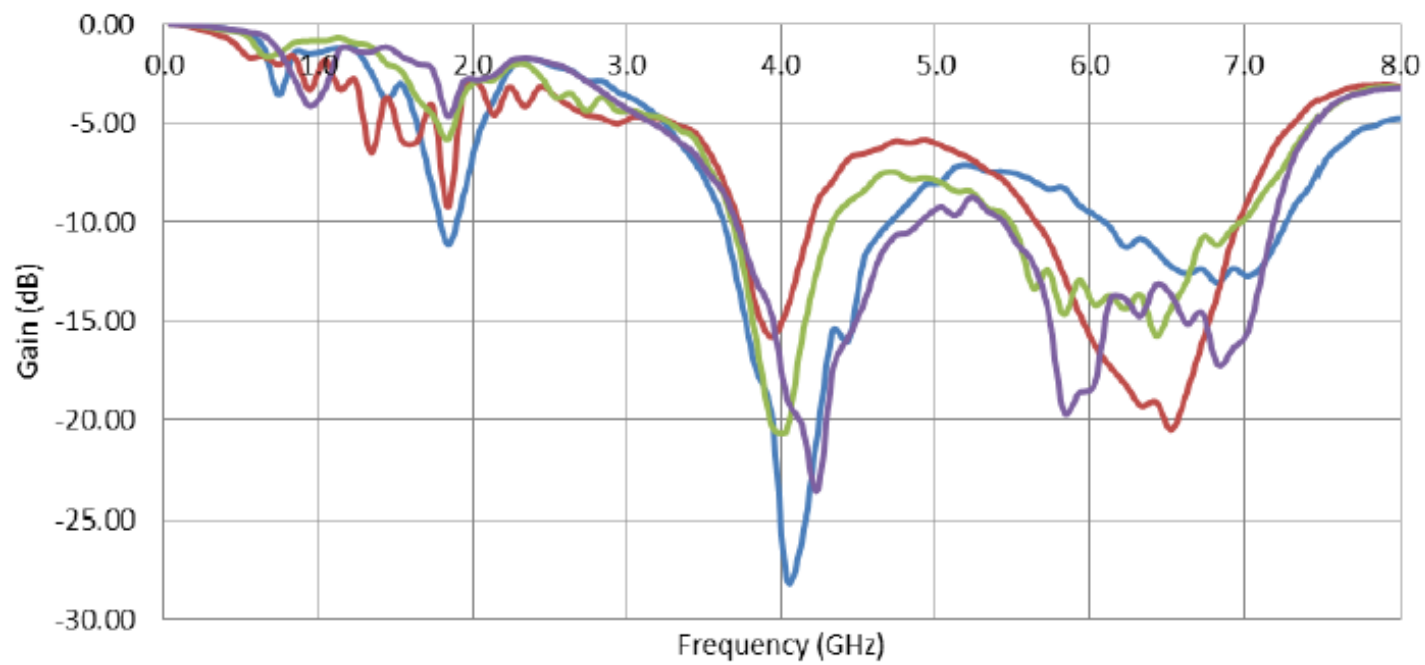

Figure 3: S11 Variation in 4 previously manufactured Vivaldi antennas.

Work by [16] indicated that the antenna response could be improved by using an antipodal design with a tapered transition from microstrip to symmetric double-sided slot-line. This removes the bandwidth limitations of the microstrip / slotline transition which means that the lower frequency limit should be solely determined by the dimension at the end of the flare. Since the electric fields across each side of the slot are no longer in a single plane, the result is a poor crosspolar performance at higher frequencies (where the geometric skew is higher) [17]. The chosen design is similar to that used in Microwave Engineering Europe's 2000 CAD benchmark comparison [17], and is based on the design in [18]. 
This design uses an offset microstrip which will skew the field, as it also does in the antipodal slot radiating part of the antenna.

The design, as given in [18], is based on a number of transmission line design calculations:

- The wavelength of the field in the material, which is a function of the permittivity of the dielectric substrate.

- The ratio of the microstrip line width and separation, which relates to the required input and output impedances and the permittivity of the substrate.

- The antenna width at the end of the flare, governed by half the largest desired wavelength in the substrate, since the waves are radiated when the edge spacing is greater than a $1 / 2$ wavelength [19].

- The length of the flare (paired stripline), which is taken to be at least 14 times the strip-line width.

Finally, the overall length of the antenna was chosen to be twice the width, so that it equalled the largest desired wavelength in the substrate.

A thicker substrate separation between top and bottom parts increases the directivity (and sidelobes), and the higher the permittivity of the substrate, the narrower the main beam [20]. However, standard thickness FR-4 is used for the substrate material since it is both low cost and easy to manufacture. FR-4 is a standard glass-reinforced epoxy laminate sheet used for printed circuit board manufacture. The dimensions and design of the chosen antenna are shown in figure 4, with the physical properties of the PCB material given in table 2.

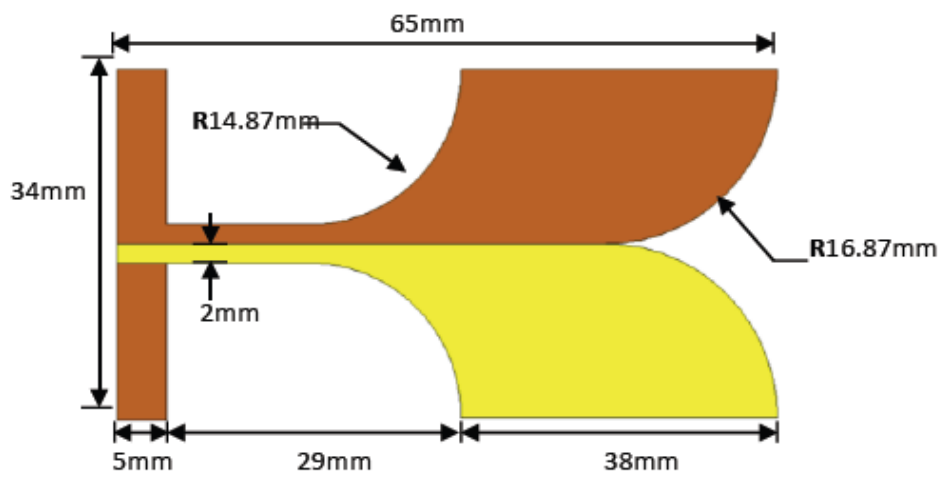

Figure 4: Antipodal Vivaldi antenna used for radar imaging.

\begin{tabular}{|l|l|}
\hline Property & Value \\
\hline Substrate relative permittivity & 4.3 \\
\hline Dielectric loss tangent & 0.018 \\
\hline Substrate thickness & $1.5 \mathrm{~mm}$ \\
\hline Copper surface thickness & $35 \mathrm{um}$ \\
\hline Substrate conductivity & $4.1 \times 10^{7} \mathrm{~S} / \mathrm{m}$ \\
\hline
\end{tabular}

Table 2: Physical properties of the PCB material.

The antenna's frequency and angular response were simulated in CST Microwave Studio and are indicated by the dashed curve in figure 5. The antennas were manufactured in the university PCB facility and measured in a microwave anechoic chamber. The experimentally measured return loss (S11) parameters are shown as the solid curve in figure 5. 


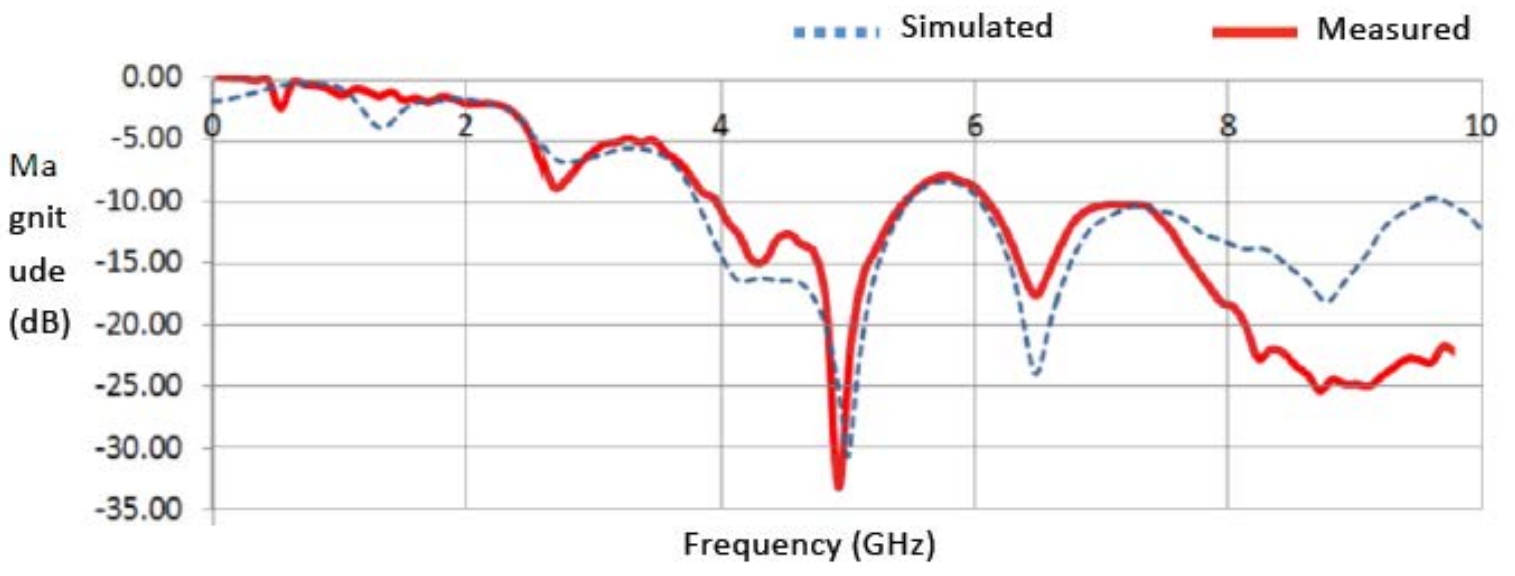

Figure 5: Antipodal Vivaldi antenna S11 response. Dashed line is simulation, solid line is measurement.

The simulation results given in [18] indicated that it should achieve a -10 dB S11 reflection coefficient between $3.3 \mathrm{GHz}$ and $6.2 \mathrm{GHz}$, with resonances at $4.5 \mathrm{GHz}$ and $5.8 \mathrm{GHz}$. However our implementation of the design in CST Microwave Studio gives a -10 dB S11 reflection coefficient between $4 \mathrm{GHz}$ and $4.7 \mathrm{GHz}$, and also above $6 \mathrm{GHz}$, with resonances at $5 \mathrm{GHz}$ and $6.3 \mathrm{GHz}$.

The E-Plane radiation pattern was measured and is shown in figure 6 below, indicating a beam-width of 85 degrees.

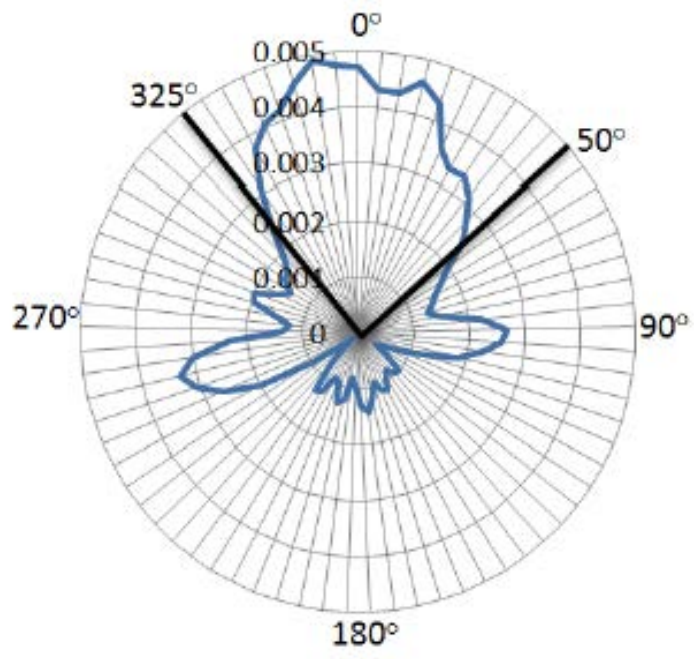

Figure 6: Antipodal Vivaldi antenna E-Plane angular radiation pattern.

The two arrays of 16 antennas were connected to the radar measurement electronics via eight multiplexers. Each multiplexer was a single pole, six throw multiport switch (Agilent L7106B). This multiplexer has the specification of an insertion loss between $-0.3 \mathrm{~dB}$ to $-0.5 \mathrm{~dB}$, and an isolation between $120 \mathrm{~dB}$ to $105 \mathrm{~dB}$ over the expected range of 1 to 10 GHz. The multiplexers were controlled using two Adlink NuDAQ PCI-7256 PCI cards and were connected together using semi-rigid copper cable with a $2 \mathrm{~dB} / \mathrm{m}$ loss. 


\section{SYSTEM CONTROL AND DATA PROCESSING}

The system control and data display was implemented in LabVIEW with the image reconstruction implemented as a MATLAB function that is called from within LabVIEW.

The data processing consisted:

- Background subtraction for the removal of antenna-cross talk and static reflections.

- Deconvolution filtering using a Wiener filter.

- Image reconstruction from the measurements of all the possible antenna pairs.

The Wiener filter requires an impulse response signal which the algorithm uses to modify the frequency response of the measurements on the unknown object. Each different antenna pair uses a separate impulse response signal. The impulse response signals are created by using a large mirror as the reflecting object.

The background signal is removed both from the impulse response signals and the object measurements as shown in figure 7.

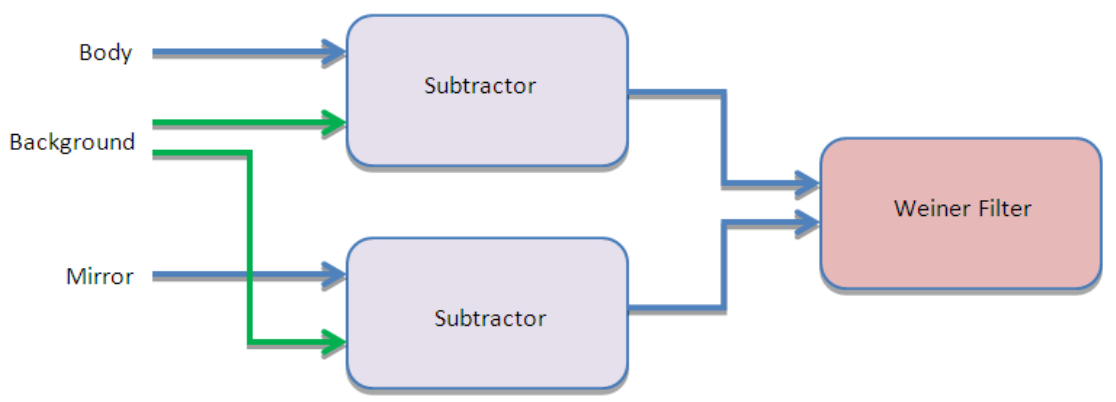

Figure 7: Data processing prior to image reconstruction.

The image reconstruction is performed through the use of the backprojection technique. The basic concept of backprojection is to transmit a pulse and record both the time taken for the subsequent reflection to reach the receiver and the corresponding magnitude of the received signal. This time taken from the transmitter to the object and back to the receiver is called the round trip time (RTT) [21].

The space in which the antennas are operating is split into cells in a grid pattern as shown in figure 8. A grid size of 450 $\mathrm{mm}$ x $500 \mathrm{~mm}$ was chosen in order to allow for flexible positioning of the human subjects. 


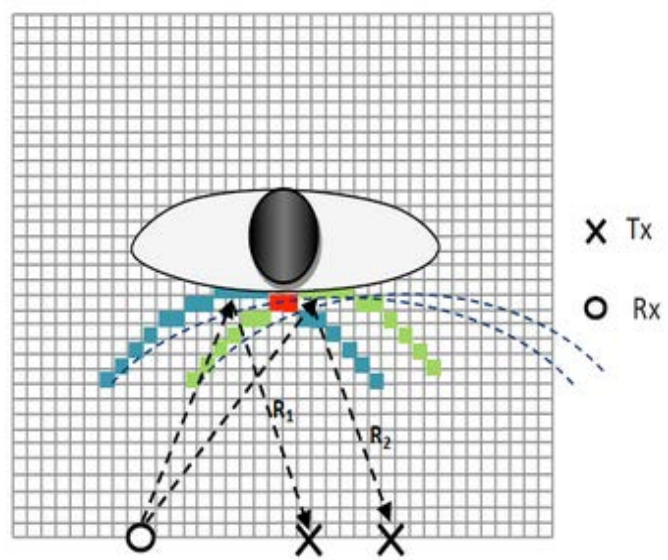

Figure 8: Image reconstruction method.

The two reflections shown in figure 8 originate from two of these cells (highlighted two cells in front of the head). However each cell does not have a unique round trip time (RTT) for a given transmitter-receiver (Tx-Rx) pair, so all the cells on an ellipse will have the same RTT. Therefore the RTT corresponding to reflection one $\left(\mathrm{R}_{1}\right)$ is not unique to a specific cell; as a result all the cells on the hyperbola are given the same magnitude. When $R_{2}$ reaches the second receiver, the RTT for this signal will not be the same as $R_{1}$, consequently this is mapped onto its own ellipse. The magnitudes of these reflection ellipses are summed. Using multiple Tx-Rx pairs and mapping their RTTs onto the corresponding ellipses, the magnitudes at the intersection points build up. This procedure produces an outline reconstruction of the object.

\section{RESULTS}

The datasets are created using a three stage process:

1. Capture background data with no object in front of the array

2. Capture the reference data by placing a large flat mirror standing perpendicular to the array, $0.6 \mathrm{~m}$ away

3. Capture the data for the object.

The two dimensional reconstruction from reflections off a $230 \mathrm{~mm}$ water bottle is shown in figure 9, along with the colour chart. The bottle has been superimposed on the image for clarity. The main section of the bottle has been clearly detected by the reconstruction algorithm.

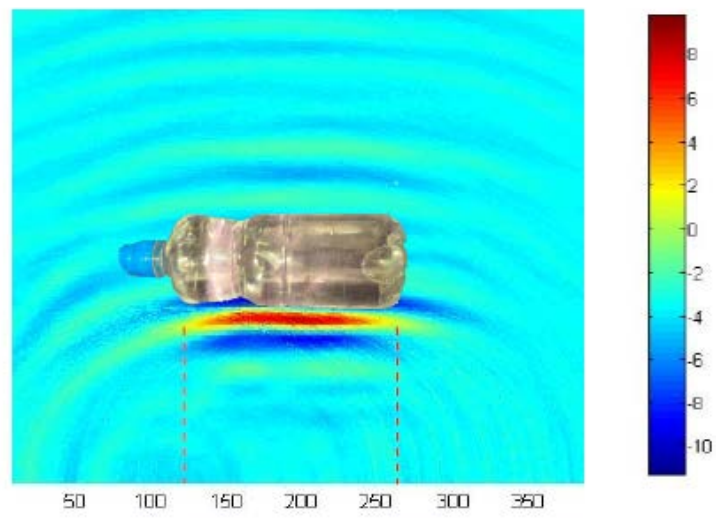

Figure 9: Image reconstruction, with the bottle overlaid. 
Figure 10 demonstrates the effect of an object placed on top of the bulk reflecting object. Here the bulk reflecting object is the water bottle and the object placed on to is a plastic rectangular solid.
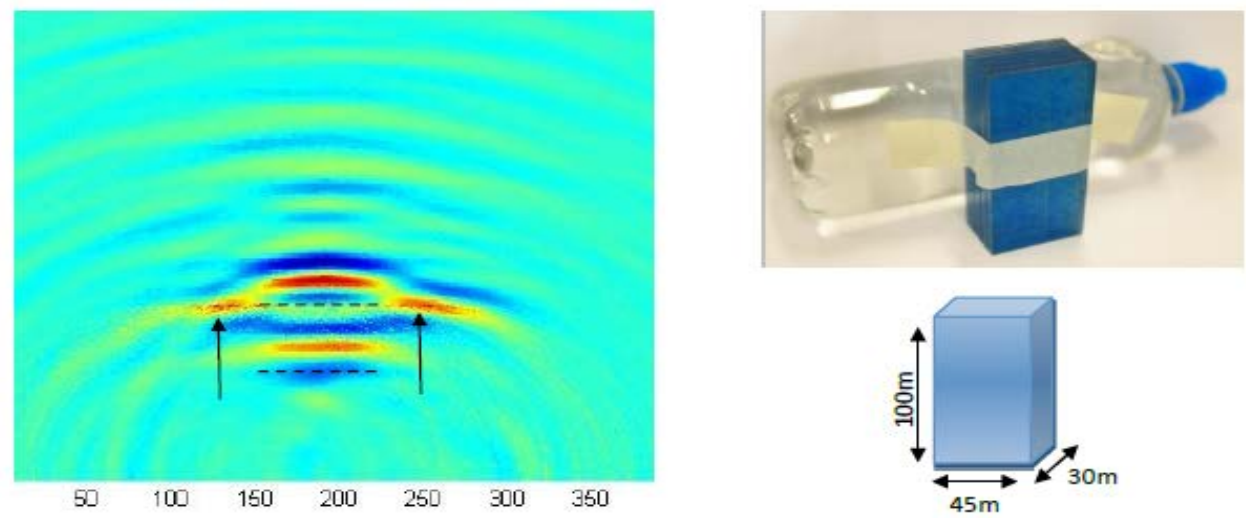

Figure 10: Rectangular solid object placed in front of the bottle and the associated UWB radar reconstruction.

The lower dashed horizontal line shown on the reconstruction in figure 10 indicates the front face of the rectangular solid object, whilst the upper dashed line is the front face of the water bottle. The back face of the rectangular solid object is coincident with the front face of the water bottle and they are shown indented into the bottle compared to reflections of the ends of the bottle. This is due to the fact that the electromagnetic waves slow down in the plastic dielectric and therefore take longer to travel the same distance relative to air. The RTT equation assumes all materials have a unity relative permittivity and therefore a constant velocity, so it maps the reflections to a position further from the antennas than they actually are, giving the impression of the indentation.

A full sized mannequin was then used as the test object to investigate the ability of the system to detect various objects placed on the human body. The mannequin was made out of polystyrene which is not as reflective as the high watercontent human body. Aluminium tape was used to cover the midsection of the mannequin in order to replicate the reflectivity of the body at these frequencies. Figure 11 shows a 2D reconstruction midway up the torso. The torso curvature can be seen, with a strong reflection from the middle region of the torso since this region is parallel to the antenna array. Similar results have been obtained from human subjects.

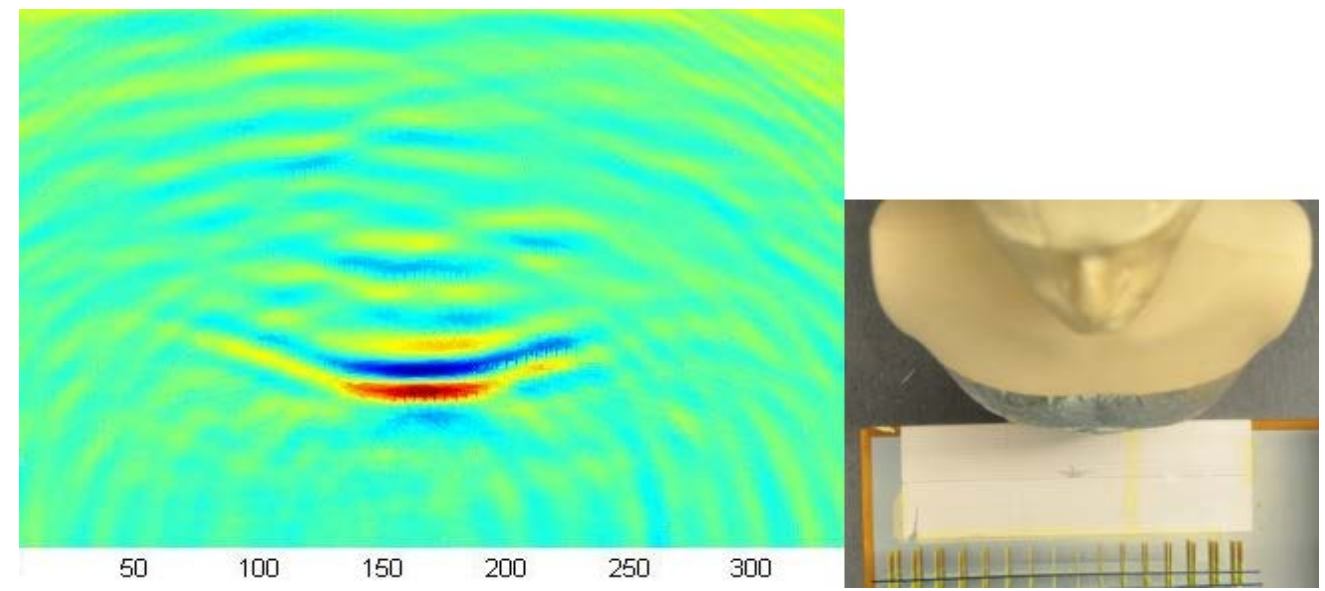

Figure 11: Midplane torso reconstruction of a mannequin. The right image is a visual plan view of the mannequin.

A plastic rectangular solid object was then placed on the torso. This produced an indent on the torso curvature in the reconstructed image, as shown in figure 12, for reasons described above. 

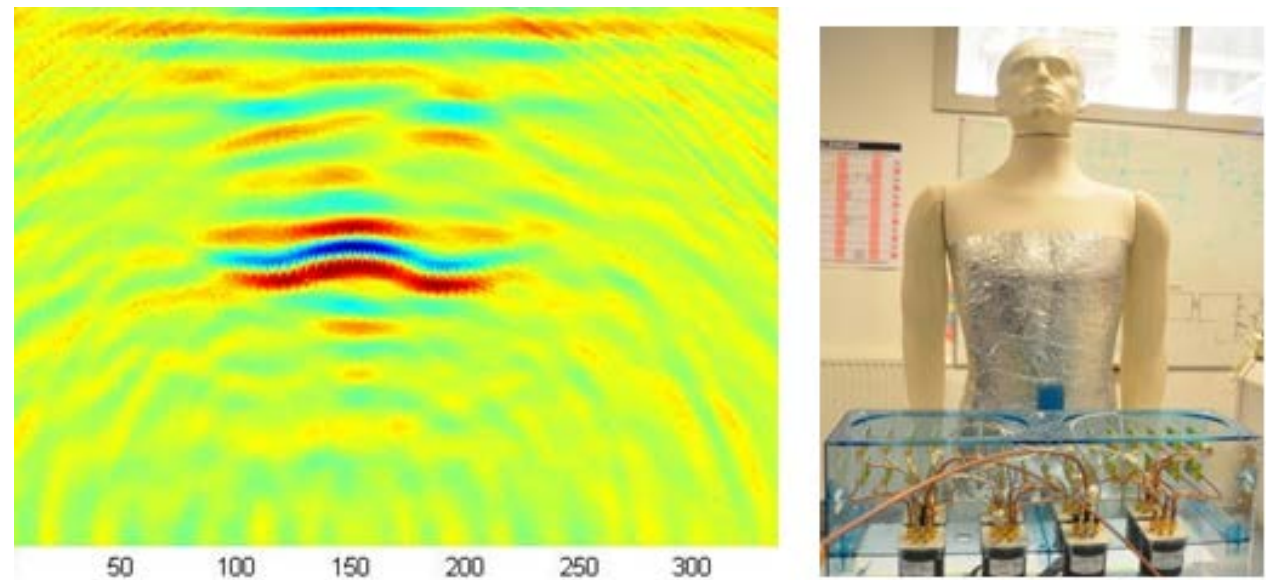

Figure 12: Reconstruction of a rectangular solid object placed in front of the mannequin. The image on the right shows the mannequin, with the top of the object visible on the centreline of its stomach, just above the imaging array.

\subsection{Extension to $3 D$ imaging}

To perform a scan of a whole torso, the linear array system was attached to a 1D scanner system. A mannequin was placed beneath the scanner as shown in figure 13. The imaging system then measured the full set of antenna pairs at ten scan positions separated by $20 \mathrm{~mm}$ intervals. The scan therefore covered the $200 \mathrm{~mm}$ torso mid-section of the mannequin.

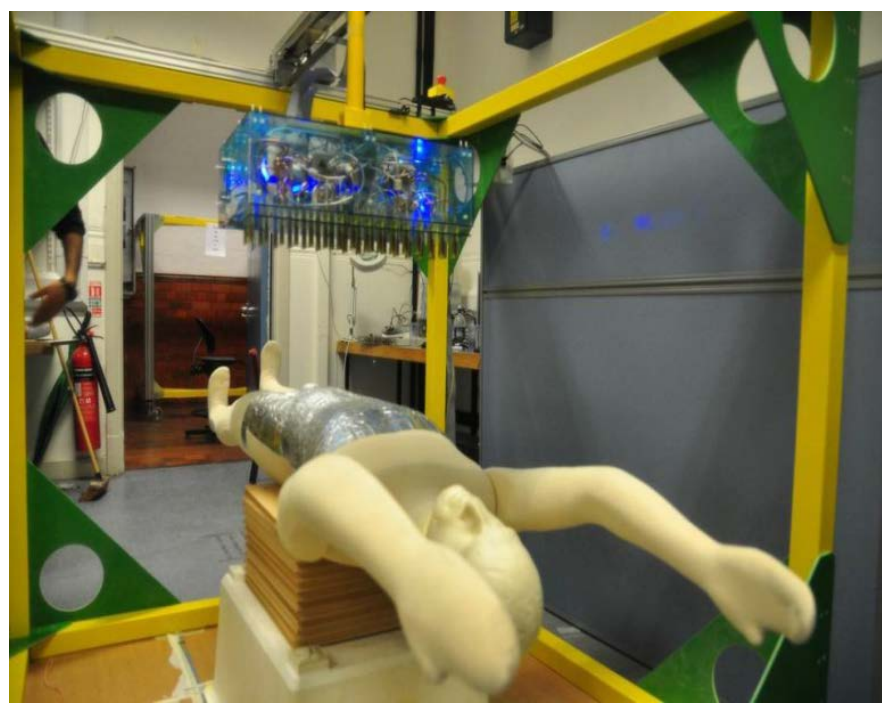

Figure 13: Mannequin placed horizontally underneath the x-y scanner.

The 2D reconstructed images were stacked using the "VolView" image display software to create a 3D image. Since only the 3D position of the surface of the object is reconstructed, the obtained reconstruction is more accurately termed a 2.5D dataset as opposed to a 3D dataset. The VolView software was also used to isolate the body signal in order to construct an image as shown in figure 14. It can be seen that the 2.5D shape gives a representation of the mannequin's torso. 


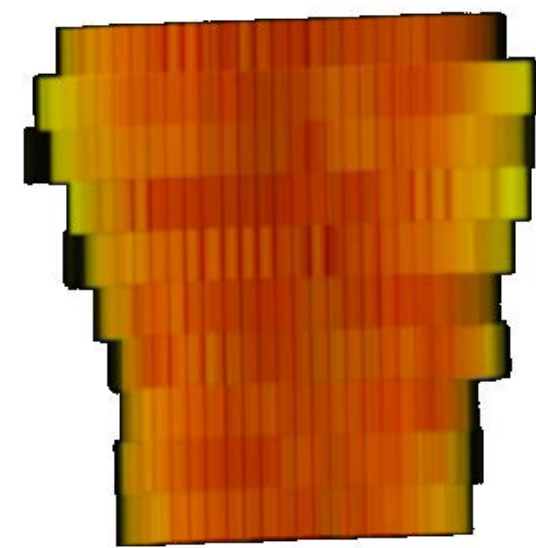

Figure 14: 2.5D reconstruction of the mannequin's torso.

A $30 \mathrm{~mm}$ x $45 \mathrm{~mm}$ x $100 \mathrm{~mm}$ plastic rectangular solid object was placed on the mannequin's torso. The resultant reconstruction can be rotated in 3D space within the software. An oblique view is shown in figure 15.

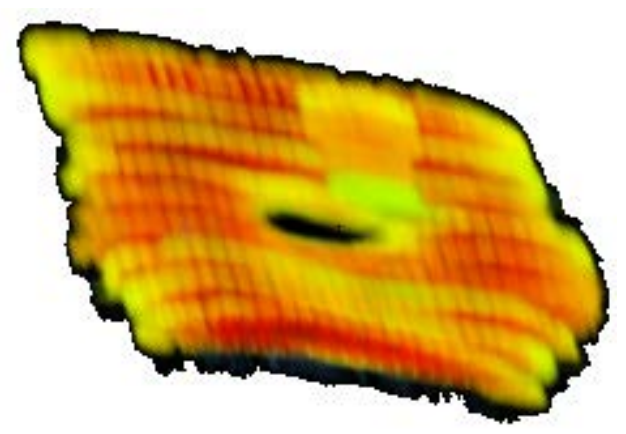

Figure 15: 2.5D reconstruction of the mannequin's torso (oblique view).

The indentation of the image caused by the object can be seen as an apparent hole in the centre of the body. A "floating" piece of material can also be seen in the image, slightly to the right and above the hole. This represents the nearest face of the rectangular solid object to the antennas, and is created by the direct reflections from the object's surface.

The minimum object size detectable on the mannequin, and also on a human body placed under a cotton t-shirt, was determined to be approximately $12 \mathrm{~mm}$ x $30 \mathrm{~mm}$ x $70 \mathrm{~mm}$.

\section{CONCLUSIONS}

This paper has described the background and requirements of whole body imaging and the context behind UWB imaging for concealed object detection. It has described the measurement system comprising separate transmit and receive linear arrays, each consisting a 16 element antipodal Vivaldi antenna array that was used for data capture.

The object surface was estimated by measuring the reflected UWB radar echoes for all possible antenna combinations of transmit and receive pairings. By scanning the antenna array over the object, a 3D surface reconstruction of the human torso was created. These reconstructed images form a convincing proof of concept.

The current system has the capability to scan and detect a concealed $12 \mathrm{~mm}$ x $30 \mathrm{~mm}$ x $70 \mathrm{~mm}$ non-metallic object placed on a human torso, under a cotton t-shirt, from a distance of $200 \mathrm{~mm}$ in 55 seconds. The authors believe that this detectability and measurement speed could be significantly improved with a better experimental system and improved image / signal processing. Therefore, the current performance does not represent the fundamental limit of the technique. 


\section{REFERENCES}

[1] Testomy Statement of R. Kane, L. Kair before the U.S. House of Representatives Committee on Oversight and Government Reform,

<http://webcache.googleusercontent.com/search?q=cache:http://www.tsa.gov/sites/default/files/publications/pdf/testimo ny/031611_tsa_kane_kair_tsa_oversight_testimony.pdf $>$

[2] Airports Council International - North America, "An Assessment of Check Point Security: House Homeland Security Subcommittee on Transportation Security and Infrastructure Protection Subcommittee", <http://www.acina.org/content/assessment-check-point-security-house-homeland-security-subcommittee-transportation-security> [3] Department of Homeland Security, Transportation Security Administration, <http://www.tsa.gov/ait-frequentlyasked-questions> Latest revision: 23 July 2013

[4] Schubert, H. and Kuznetsov, A., "Detection and Disposal of Improvised Explosives", 2006, Springer, section 6.4 Terahertz

[5] Ellison, W.J., "Permittivity of Pure Water, at Standard Atmospheric Pressure, over the Frequency Range 0-25 THz and the Temperature Range 0-100 ${ }^{\circ} \mathrm{C}$ ", J. Phys. Chem. Ref. Data, Vol. 36, No. 1, 2007, DOI: 10.1063/1.2360986 [6] Fernandes, J.L., Rappaport, C.M. and Sheen, D.M., "Improved reconstruction and sensing techniques for personnel screening in three-dimensional cylindrical millimeter-wave portal scanning”, Proc. SPIE 8022, Passive Millimeter-Wave Imaging Technology XIV, 802205 (May 25, 2011); doi:10.1117/12.887612.

[7] Department of Homeland Security, Office of the Director General, November 2011, “TSA Penetration Testing of Advanced Imaging Technology: Unclassified Summary ”, <http://www.oig.dhs.gov/assets/Mgmt/OIG_SLR_1206_Nov11.pdf>

[8] European Commission IP/11/1343, “Aviation security: Commission adopts new rules on the use of security scanners at European airports”, 14/11/2011, <http://europa.eu/rapid/press-release_IP-11-

1343_en.htm?locale=en\#PR_metaPressRelease_bottom>

[9] Kravets, D., “TSA Pulls Plug on Airport Nude Body Scanners”, Wired on-line magazine, 01/18/13

$<$ http://www.wired.com/threatlevel/2013/01/tsa-abandons-nude-scanners/>

[10] Wild, N.C., Doft, F., Breuner, D. and Fe1ber F., "Handheld ultrasonic concealed weapon detector", in "Enabling Technologies for Law Enforcement and Security", Proc. SPIE Vol. 4232 (2001)

[11] Taylor, J.D., [Introduction to Ultra-Wideband Radar Systems], CRC Press, 1995, ISBN: 0849344409

[12] Guo, L., "Study and Miniaturisation of Antennas for Ultra Wideband Communication Systems", PhD thesis 2009,

School of Electronic Engineering and Computer Science, Queen Mary, University of London, UK

[13] iDa Singapore, "Technical Specification for Ultra Wideband (UWB) Devices", IDA TS UWB: 2007, Info-

Communications Development Authority of Singapore, Resource Management \& Standards.

[14] Fannjiang, A.C. and Sølna, K., "Broadband resolution analysis for imaging with measurement noise", JOSA A, Vol.

24, Issue 6, pp. 1623-1632 (2007), <http://dx.doi.org/10.1364/JOSAA.24.001623>

[15] Harmer, S.W, Andrews, D.A., Rezgui, N.D. and Bowring, N.J., "Detection of handguns by their complex natural resonant frequencies”, IET Microw. Antennas Propag., 2010, Vol. 4, Iss. 9, pp. 1182- 1190, doi: 10.1049/ietmap.2009.0382

[16] Gazit, E., “Improved design of the Vivaldi antenna”, IEE Proc. Vol. 135, Pt. H, No. 2, April 1988.

[17] Microwave Engineering Europe, “The 2000 CAD Benchmark unveiled”, November 2000,

<http://i.cmpnet.com/edtn/europe/mwee/pdf/CAD.pdf>

[18] Mohammad, N.H. and Ismail, W., "System-level Integration and Simulation of Ultra Wideband Receiver Front-

End”, Communications, Propagation and Electronics, MIC-CPE, 2008 Mosharaka International Conference. DOI:

10.1109/MICCPE.2008.4555755

[19] Maalik, S., “Antenna Design for UWB Radar Detection Application”, Master of Science Thesis, Communication Engineering, Chalmers University of Technology, 2010.

[20] Lee, R.Q., “Notch Antennas”, in Encyclopedia of RF and Microwave Engineering, Wiley, 2005. DOI:

10.1002/0471654507.eme297

[21] Zeng, G.L., [Medical Image Reconstruction: A Conceptual Tutorial], Springer, Higher Education Press, 2010. ISBN 3642053688 . 